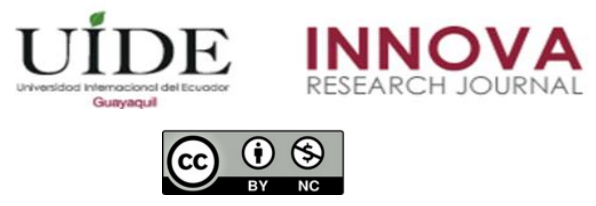

INNOVA Research Journal, ISSN 2477-9024

(Enero-Abril 2020). Vol. 5, No.1 pp. 234-241

DOI: https://doi.org/10.33890/innova.v5.n1.2020.1278

URL: http://revistas.uide.edu.ec/index.php/innova/index

Correo: innova@uide.edu.ec

\title{
Las tasas para la obtención de documentos de control previo en Ecuador como medidas para restringir las importaciones
}

\section{Fees for obtaining prior control documents in Ecuador as measures to restrict imports}

\author{
Edgar Vicente Larco Camacho \\ Universidad Internacional del Ecuador, Ecuador \\ Evelin Bazurto Campuzano \\ Universidad Internacional del Ecuador, Ecuador
}

Autor por correspondencia: edlarcoca@uide.edu.ec; evbazurtoca@uide.edu.ec

Fecha de recepción: 20 de diciembre del 2019 - Fecha de aceptación: 09 de enero del 2020

\section{Resumen}

Las tasas a las importaciones afectan al consumidor ecuatoriano. El presente estudio busca analizar las tarifas que los importadores cancelan a las instituciones que emiten los permisos para las importaciones. La información obtenida derivó del análisis y descripción de la información de las agencias de control y entrevistas a importadores del Ecuador. Los resultados permiten ver que las tarifas a cancelar para obtener los documentos de control previo, tienen valores variables conforme el tipo de requisito y la institución que lo emite. El estudio también visibiliza los valores de las tasas de las instituciones de control y detalla la cuantía a cancelar al Instituto Ecuatoriano de Normalización INEN por Certificado de Reconocimiento, que se sitúa en un promedio de \$247,00. Las tarifas que se cancelan a la Agencia de Regulación, Control y Vigilancia Sanitaria ARCSA para productos extranjeros varían de $\$ 542,12$ a $\$ 2.258,41$; los Permisos de Funcionamiento de AGROCALIDAD y el Registro de Productos fertilizantes son de $\$ 1048,58$ y $\$ 560,95$ respectivamente. Se concluye que las tasas limitan a los nuevos y pequeños importadores en sus actividades comerciales y encarecen los precios de los productos importados.

Palabras clave: documentos de control previo; importaciones; tasas por servicios; restricciones de importación

\begin{abstract}
Import rates affect Ecuadorian consumers. The present study seeks to analyze the tariffs that importers pay to the institutions that issue permits for imports. The information obtained was derived from the analysis and description of the information of the control agencies and interviews with importers from Ecuador. The results show that the rates to be canceled to obtain the prior control documents have variable values according to the type of requirement and the institution that issues it. The study also makes visible the values of the rates of the control institutions and details the amount to cancel the Ecuadorian Institute of Standardization INEN for Certificate of Recognition, which stands at an average of \$247.00. The rates that are paid to
\end{abstract}


the ARCSA Health Regulation, Control and Surveillance Agency for foreign products vary from \$ 542.12 to \$2,258.41; AGROCALIDAD Operating Permits and the Register of Fertilizer Products are $\$ 1048.58$ and $\$ 560.95$ respectively. It is concluded that the rates limit new and small importers in their commercial activities and increase the prices of imported products.

Key words: prior control documents; imports; service fees; import restrictions

\section{Introducción}

El gobierno del Ecuador puso en ejecución una serie de medidas con el objetivo de restringir las importaciones. Un ejemplo de estas medidas son la sobretasa arancelaria implementadas en el 2015, los cupos a las importaciones en el 2012, y la actualización del listado de productos que requieren certificado de reconocimiento INEN en el 2013. Estas medidas ayudaron a reducir en las importaciones (Izaguirre, Bazurto y Plúa, 2019, pp. 66-67). Entre otras medidas también desempeñan un rol muy importante los documentos de control previo que deben tramitar los importadores antes de la importación de sus productos y que deben ser presentados como documentos de acompañamiento en la Declaración de Aduanera de Importación (Asamblea Nacional, 2017, pp. 17-18). Y su importancia se debe a que los costos que representa obtener los permisos pueden ser relativamente altos.

Las tasas de los permisos y certificados que se deben obtener antes de la importación de los productos que los requieren, según el tipo de producto y la partida arancelaria, son una limitante al momento de iniciar los trámites para los pequeños o nuevos importadores. Los documentos como el Registro Sanitario y Notificación Sanitaria que son emitidos por la Agencia de Regulación, Control y Vigilancia Sanitario (ARCSA) para alimentos procesados, medicamentos, productos naturales de uso medicinal, maquillaje, productos higiénicos de uso industrial. El Certificado de reconocimiento otorgado por el Instituto Ecuatoriano de Normalización INEN para una gran variedad de productos. El Permiso de Importación otorgado por el Ministerio de Agricultura, Pesca y Acuacultura MAPA para productos de la industria agrícola, acuícola y pesquera.

En un informe presentado por la Cámara de Comercio de Guayaquil (2019), indica que el cambio propuesto por el INEN sobre la eliminación de los "Certificados de primera parte” por "Certificado de conformidad de producto" representaría un incremento de los precios finales de los productos debido a la tramitología, además que el costo del Reglamento Técnico se incrementaría hasta en un 1,600\%. Por lo tanto, la Cámara de Comercio de Guayaquil planteó una propuesta para "reformar, eliminar o permitir la homologación" de los certificados, además proponen la reducción de costos del Certificado INEN-1 y su ampliación en el plazo de validez. Con esta propuesta se pretende reducir costos y tiempos en el proceso para obtener el certificado INEN, además que el precio final de los productos no sea afectado para los consumidores.

En el 2013 el Pleno del COMEX puso en ejecución la Resolución 116 donde se reformó la nómina de los productos que están sujetos a controles previos a la importación y que deben presentar el Certificado de Reconocimiento INEN. Este Certificado se exige como documento de soporte a la Declaración de Importación. Esta medida incrementó las partidas arancelarias que deben cumplir la presentación del Certificado de Reconocimiento para regular el ingreso los productos a territorio ecuatoriano (COMEX, 2013). Por otra parte, en el 2018 el COMEX derogó

Esta obra se comparte bajo la licencia Creative Common Atribución-No Comercial 4.0 International (CC BY-NC 4.0) Revista de la Universidad Internacional del Ecuador. URL: https://www.uide.edu.ec/ 
la resolución 116-2013 mediante Resolución 24-2018 en la que se excluyen a más de doscientas subpartidas arancelarias de la presentación del Certificado de Reconocimiento (COMEX, 2018).

En la tabla 1 se muestra la variación de las importaciones analizando el mismo periodo trimestral comparando las importaciones de los dos primeros semestres de los años 2017 y 2018 con los dos primeros semestres del año 2019 posterior a la emisión de la resolución 24-2018 del COMEX.

Tabla 1.

Variación en las importación ler y 2do semestre, años 2017, 2018 y 2019

\begin{tabular}{|c|c|c|c|c|c|c|c|c|}
\hline \multirow{2}{*}{ Período } & & \multirow{2}{*}{2017} & \multirow{2}{*}{\multicolumn{2}{|c|}{2018}} & \multirow{2}{*}{\multicolumn{2}{|c|}{2019}} & \multicolumn{2}{|c|}{ Variación } \\
\hline & & & & & & & 2019 vs 2017 & 2019 vs 2018 \\
\hline 1er Trimestre & $\$$ & $4.321,86$ & $\$$ & $5.075,84$ & $\$$ & $5.365,23$ & $24 \%$ & $6 \%$ \\
\hline 2do Semestre & $\$$ & $4.626,68$ & $\$$ & $5.572,87$ & $\$$ & $5.715,93$ & $24 \%$ & $3 \%$ \\
\hline
\end{tabular}

Fuente: Banco Central del Ecuador

Como se muestra en la tabla 1 las importaciones en los dos primeros trimestres del año 2019 crecieron significativamente con respecto al año 2017, y la variación con respecto al año 2018 fue de $6 \%$ y $3 \%$ respectivamente. En la tabla no se muestra un impacto significativo de las importaciones del 2019 vs 2018, luego de la actualización de la nómina de subpartidas arancelarias que no deben presentar el certificado de reconocimiento INEN-1.

\section{Metodología}

Para realizar el estudio se implementó una metodología descriptiva. Se obtuvo el valor de las tasas de Servicios o Permisos que se requieren para las importaciones de las instituciones públicas correspondientes a cada documento de control. Dichos valores se consultaron en la página del INEN para los Certificados de Conformidad con el Formulario INEN-1. En la página de ARCSA para las tasas por los Registros Sanitarios y las Notificaciones Sanitarias y en la página de AGROCALIDAD.

Además, para complementar el estudio se realizó entrevistas a diferentes importadores que este tipo de permisos para ingresar sus productos al país.

\section{Revisión de la literatura}

En el Art 72 del Reglamento al Libro V del COPCI define que los documentos de acompañamientos, que son presentados como un requisito en la Declaración Aduanera de Importación, son los denominados documentos de control previo. Estos documentos deben ser tramitados y aprobados antes que las mercancías sean embarcadas para la importación. (Asamblea Nacional, 2017)

Según un estudio realizado por Solano (2019) analizó el efecto que tiene la emisión del certificado como documentos de control previos electrónico emitido por Agrocalidad y el procedimiento para importar los medicamentos veterinarios. En su estudio Realizó una 
investigación documental, además empleó la técnica de observación y la encuesta a importadores en Guayaquil con el objetivo de determinar el impacto de los documentos de control previo sobre las importaciones y concluyó que el tiempo en obtener la licencia electrónica mejoró los tiempos en comparación con las licencias físicas, pero también destaca que los procesos se vieron entorpecidos por el proceso digital retrasando las operaciones de importación.

Por su parte Torres y Velasco (2017) desarrollaron un estudio que tiene como objetivo analizar la incidencia en los tiempos de gestión y costos de los permisos de importación de INEN y Agrocalidad sobre la cadena de logística de Comercio Exterior. Realizó un estudio cuantitativo y utilizó una metodología descriptiva y analítica donde se concluyó que los lineamientos para los tramites de las entidades de INEN y Agrocalidad no son viables para muchos de los importadores provocando que existan costos por re-procesos que deben ser asumidos por los importadores, por lo tanto, también los consumidores son afectados por los precios de los productos.

Para tener otra perspectiva Baena (2018) analizó el proteccionismo en el comercio internacional. El autor hizo una investigación tomando información de fuentes secundarias como el Ministerio de Comercio Exterior, la Organización Mundial del Comercio y el Global Trade Alert y desarrolló un estudio descriptivo que permitió medir indicares referentes a las Barreras Arancelarias y No Arancelarias. Donde obtuvo como resultados que las Barreras No Arancelarias superan a las Barreras Arancelarias como medidas de proteccionismo, tomando en cuenta que las Barreras No Arancelarias han aumentado y que en economías en desarrollo son en promedio más altas.

Asimismo, Barajas (2019), analizó las barreras no arancelarias aplicadas en Chile como un obstáculo para las exportaciones colombianas, considerando que las barreras arancelarias disminuyen mientras que las barreras no arancelarias aumentan como medida de protección. En su estudio el autor realizó un análisis cualitativo donde hizo una comparación de las medidas para determinar cuáles eran los obstáculos de las exportaciones colombianas. El autor concluyó que las exportaciones que realiza Colombia tienen mayores dificultades al momento de comercializar sus productos hacia el mercado chileno debido a los registros, documentación y licencias que Chile exige en sus importaciones. Además, las nuevas medidas proteccionistas internas de chile y las demoras en procesos de certificación son un obstáculo más para los exportadores colombianos.

De igual manera, Gil (2015), define en su artículo acerca de las restricciones o las barreras considerando las restricciones no arancelarias como un problema para la MERCOSUR. El autor realizó un análisis de la legitimidad de las medidas que implementan los países como restricciones no arancelarias. El autor concluye que las restricciones no arancelarias se dificultan al ser identificadas y definidas, por lo tanto representan un problema común del comercio internacional y regional, lo que a su vez puede ser una lección política que permite a los tribunales a dar distintas interpretaciones sobre la legitimidad o ilegitimidad para definir e identificar de las restricciones no arancelarias.

Finalmente Baena y Cano (2018) presentaron un análisis sobre las restricciones en el comercio internacional sobre las exportaciones como una política comercial bajo el marco del 
GATT y de la OMC. En su estudio identificaron el planteamiento de disputas sobre las medidas arancelarias y medidas no arancelarias que se aplican a las exportaciones de bienes. Los autores concluyen que existe la necesidad de que la OMC desarrolle nuevas políticas que regulen las restricciones al comercio internacional de forma más adecuada.

Los controles que realizan las instituciones gubernamentales en las importaciones limitan el ingreso de los productos regulados. Al ser medidas no arancelarias, los documentos de control son una barrera al comercio exterior.

\section{Resultados}

\section{Tarifas por tipo de documento de control previo}

En las tablas que se presentan a continuación se detallan los valores de las tasas que cobra cada institución por certificados, permisos y registros. Se presentan de forma individual las entidades regulatorias con los respectivos valores por los trámites y documentos.

Tabla 2.

Tasas Registros Sanitarios/Notificaciones Sanitarias

\begin{tabular}{|c|c|}
\hline Servicio & Tasa \$ \\
\hline Medicamentos Extranjeros & $\$ 2.258,41$ \\
\hline Medicamentos homeopáticos extranjeros & $\$ 904,34$ \\
\hline Dispositivos médicos extranjeros & $\$ 904,34$ \\
\hline Alimentos procesados extranjeros & $\$ 904,34$ \\
\hline $\begin{array}{l}\text { "Productos cosméticos extranjeros (varios titulares con un mismo código de la notificación sanitaria } \\
\text { obligatoria, pagara en forma individual)" }\end{array}$ & $\$ 904,34$ \\
\hline Productos para higiene de uso doméstico y absorbentes de uso personal - extranjeros & $\$ 904,34$ \\
\hline Productos para higiene de uso industrial- extranjeros & $\$ 904,34$ \\
\hline Medicamentos Genéricos Extranjeros & $\$ 565,21$ \\
\hline Plaguicidas de uso doméstico extranjero & $\$ 542,12$ \\
\hline
\end{tabular}

Fuente: Agencia de Regulación, Control y Vigilancia Sanitaria (2019)

Los documentos de control previo que se tramiten con el ARCSA tienen diferentes tarifas que varían según el tipo de producto. Si se comparan los valores de permisos para importación con los permisos para producción nacional, las tasas por los documentos de control previo son más altas. Se debe considerar además que para gestionar los registros o notificaciones sanitarias, los importadores deben contar con la firma de aprobación de un químico farmacéutico, gestión que puede crear demoras en los trámites.

Tabla 3.

Costos por Certificados de reconocimiento INEN

\begin{tabular}{lc}
\hline \multicolumn{1}{c}{ Servicio } & Valor $\mathbf{\$}$ \\
\hline Certificado de reconocimiento de conformidad con Reglamento & 247,00 \\
Certificado de productos no sujeto a reglamento técnico & 247,00 \\
Oficio de evaluación de informes de vehículos & 85,00 \\
\hline
\end{tabular}

Fuente: Instituto Ecuatoriano de Normalización.

Para reducir los costos se requiere generar mayor eficiencia en los procedimientos para obtener los documentos de control previo. Un ejemplo de esto son los trámites del Certificado de 
Reconocimiento INEN, este certificado puede ser tramitado también cuando la mercancía llega a territorio ecuatoriano. Una mercancía que ya se encuentra en los almacenes temporales de los puertos o el aeropuerto comienza a pagar el bodegaje correspondiente desde el momento que ingresan al almacén. Si el Técnico Operador de la Aduana sugiere el cambio de la partida arancelaria, y ésta requiere certificado de reconocimiento INEN-1, el importar tiene la libertad de tramitar el certificado. En el tiempo que le tome al importador obtener el certificado, su mercancía cancelará el valor de almacenaje. Tomando como ejemplo este caso, mientras se gestione el Certificado INEN en menor tiempo, menos cancelará por bodegaje el importador al almacén temporal, caso contrario, los valores por almacenaje sumarán cada día que se demore en obtener el certificado y nacionalizar la mercancía.

Tabla 4.

Costos por permisos de funcionamiento de Agrocalidad

\begin{tabular}{lc}
\multicolumn{1}{c}{ Certificados } & Tasa \$ \\
\hline Registro, ampliación de actividad de personas naturales o jurídicas como: & 1048,58 \\
fabricantes, formuladores, envasadores, importadores, exportadores, & \\
distribuidores, aplicadores aéreos y terrestres de plaguicidas y productos afines de & \\
uso agrícola. & \\
\hline
\end{tabular}

Fuente: Agencia de Regulación y Control Fito y Zoosanitario (2019).

Los permisos que deben obtener las empresas que importan y comercializan insumos para sector agropecuario deben realizar diferentes trámites, cada trámite tiene un valor distinto. Entre los valores que deben cubrir son las tasas por funcionamiento de las instalaciones, la autorización para importar y el registro de los productos a importar. En la tabla 4 se presentan el valor de la tasa que debe cancelar el importador para obtener el permiso para ejercer.

Tabla 5.

Costos por registro de productos fertilizantes de Agrocalidad

\begin{tabular}{lc}
\multicolumn{1}{c}{ Certificados } & Tasa \$ \\
\hline Registro, ampliación de actividades de personas naturales o jurídicas como: & 560,95 \\
fabricante, formulador, envasador, distribuidor (cuando tenga más de 1 actividad & \\
aplica d. Grande), exportador, importador de fertilizantes, enmiendas de suelo y \\
productos afines de uso agrícola
\end{tabular}

Fuente: Agencia de Regulación y Control Fito y Zoosanitario (2019).

Los importadores deben cancelar a Agrocalidad la tasa que se muestra en la tabla 5 para el registro de los productos fertilizantes que van importar. Asimismo, para el registro de un nuevo producto los importadores deben gestionar con la entidad los permisos para la nueva importación.

\section{Entrevistas}

En una entrevista realizada a Suárez (2019), copropietario de importadora y comercializadora de productos cosméticos extranjeros, indicó que los valores que tuvo que cancelar al ARCSA por las notificaciones sanitarias para las importaciones de sus productos sí fueron altos. Los pagos fueron realizados por cada una de las Notificaciones correspondientes a cada producto a importar y el desembolso por cada Notificación Sanitaria fue de 904,34 dólares. 
En la entrevista dio a conocer que la empresa contaba con el capital que requería invertir para su funcionamiento, los permisos para importar, entre otros. Además, mencionó que, para un pequeño importador, estas tarifas para obtener las notificaciones sanitarias le hubieran sido un impedimento para importar los cosméticos.

Por otra parte se entrevistó a Vásquez (2019), quien importa equipos para barcos bajo la razón social de Navelec S.A., de los cuales los artículos que requieren INEN son cables y detectores de humos. En sus trámites, la empresa NAVILEC gestiona los trámites del certificado de reconocimiento con los agentes de aduana previo al embarque de su mercancía para no tener demora en un su proceso de importación y nacionalización. Vásquez indicó que los valores que debe cancelar al INEN por el certificado de reconocimiento sí influye directamente en el costo de su importación y finalmente el precio que cancela sus clientes.

\section{Conclusión}

En Ecuador el proteccionismo y la restricción a las importaciones fueron en aumento en la última década. Los documentos de control previo han sido requisitos importantes en los trámites de importación, incidiendo en los costos y restringiendo en las operaciones de los pequeños importadores.

Es importante comprender que los costos que cobran las instituciones gubernamentales para otorgar los certificados de calidad influyen directamente en el costo final de los productos. En el caso de los permisos para importar insumos agrícolas, los valores que deben cancelar para el registro de productos fertilizantes influyen en la producción agrícola, de forma más directa para aquellos productores que requieren de fertilizantes que no se producen nacionalmente. Cabe mencionar que estos permisos certifican la calidad de los insumos agrícolas a importar, de no ser así, los productos fertilizantes que ingresen sin control corren el riesgo de dañar producción agrícola.

De la misma forma, el control que se realiza sobre el ingreso de los productos extranjeros que son regulados por el ARCSA, también certifican su calidad para el consumo, y a su vez, la tasa del documento de control previo son más altas en comparación con los permisos para la producción nacional. Son necesarios los controles que se deben realizar a los productos mencionados. Pero además debe existir un equilibrio en el costo de los certificados de calidad y el tiempo poder obtener los permisos. Una elevada tarifa y demoras en los procesos para obtener un documento de control previo también limita las importaciones al país, por lo que se consideran como una medida restrictiva para las importaciones, siendo de carácter no arancelario.

\section{Bibliografía}

Agencia de Regulación y Control Fito y Zoosanitario. (31 de Marzo de 2019). Tarifario 2019.01. Agencia de Regulación, Control y Vigilancia Sanitaria. (2019). Tasas vigentes 2019.

Asamblea Nacional. (Diciembre de 2017). Reglamento al Libro V del COPCI. Documentos de Acompañamiento, 17, 18. 
Baena Rojas., J. J. (2018). Barreras arancelarias y no arancelarias como restricciones al comercio internacional. Revista Venezolana de Gerencia, , 3, 8, 11, 13.

Banco Central del Ecuador. (2019). Bienes. Boletín No 68 - 2002. I Trimestre - 2019. II Trimestre.

Banea Rojas, J., \& Cano, J. (2018). Analysis of export restraints disputes as trade policy within the GATT and WTO regime. Innovation Management and Education Excellence through Vision 2020, 2674.

Barajas Ramírez, A. M. (2017). Restricciones no arancelarias al comercio chileno: principales obstáculos para los exportadores colombianos. Revista Logos Ciencia y Tecnología, 107110.

Cámara de Comercio de Guayaquil. (2019). La tramitología Inenficiente contraataca. El comercio seguirá lento si se aumentn las trabas. . Guayaquil.

Comité de Comercio Exterior. (Noviembre de 2013). Resolución 116-2013.

Comité de Comercio Exterior. (Noviembre de 2018). Resolución 24-2018.

Gil, L. (2015). Las restricciones no arancelarias argentinas en el MERCOSUR: normas y política en los conflictos comerciales. Cuadernos de Política Exterior Argentina, 8-30.

Instituto Ecuatoriano de Normalización. (s.f.). Costos por Servicios. Recuperado el Abril de 2019, de http://www.normalizacion.gob.ec/costos-por-servicios/

Izaguirre, J., Bazurto, E., \& Plúa, C. (2019). Impacto de la Sobretasa Arancelaria Implementada en el 2015 sobre el Nivel de Importaciones y el Pleno Empleo en el Ecuador. Innova Research Journal, 66-67.

Solano Tomala, C. (2017). Repositorio Universidad de Guayaquil. Recuperado el 2019, de El documento de control previo electrónico en las importaciones de medicamentos veterinarios: http://repositorio.ug.edu.ec/bitstream/redug/18222/1/TESIS.pdf

Suárez Checa, R. (Junio de 2019). Las tarifas de ARCSA por la Notificación Sanitaria para productos cosméticos. (E. Bazurto Campuzano, Entrevistador)

Torres, J., \& Velasco, L. (Marzo de 2017). Repositorio Universidad de Guayaquil. Obtenido de Análisis de los permisos de importación INEN-Agrocalidad y su incidencia en la gestión de Comercio Exterior.

Vásquez, E. (Diciembre de 2019). Tarifas por certificado de reconocimiento INEN. (E. Bazurto, Entrevistador) 